\title{
Implante auditivo de tronco cerebral en niños
}

\author{
Auditory brainstem implant in children
}

Prof. Dr. Vicente Guillermo Diamante, Norma Pallares (MA) ${ }^{1}$

\begin{abstract}
RESUMEN
Objetivo: Mostrar los resultados obtenidos mediante el uso del Implante Auditivo de Tronco Cerebral (IATC) en niños con imposibilidad de recibir un implante coclear (IC), con hipoacusia no asociada a Neurofibromatosis tipo II (NAI). Tipo de estudio: Presentación de casos.

Material y método: Se muestran los resultados obtenidos en los dos primeros casos en América en niños (3 y 10 años, respectivamente) a los que se les colocó un IATC por agenesia de nervio y cóclea.

Resultados: Se logró niveles de 30db a 40 db en las audiometrías posteriores a la activación en frecuencias de 250-6.000 Hz, lograron detectar el test de Ling en su totalidad, con buena y rápida respuesta al nombre en una situación estructurada y sin pistas visuales dentro del primer mes de activación.

Conclusiones: De acuerdo con nuestra reciente experiencia los casos de niños con agenesia de cóclea y/o nervio, pueden ser considerados para la colocación de un IATC porque la anatomía de los núcleos auditivos está intacta.

Palabras clave: Implante Auditivo de Tronco Cerebral, implante coclear, agenesia de cóclea, malformación coclear, hipoacusia severa.
\end{abstract}

\section{ABSTRACT}

Aim: To discuss the results obtained with auditory brainstem implant (ABI) in children non-eligible for cochlear implant, presenting with non-NFI related hypoacusia. A case report study.

Materials and Method: Two cases are presented, both of children (aged 3 and 10 years old, respectively), who underwent $A B I$ because of nerve and cochlear agenesis

Results: Post-implant audiometric levels were 30 to $40 \mathrm{~dB}$ for frequencies between 25 and $6000 \mathrm{~Hz}$ Both patients were able to fully detect the Ling sound test with good and fast answers in a structured non-visual cues-situation within the first post-activation month.

Conclusions: In our experience, children with nerve and/or cochlear agenesis could be considered for ABS implantation, because the auditory nuclei's anatomy is intact.

Key words: Brainstem auditory implant, cochlear implant, cochlear agenesis, cochlear malformation, severe hypoacusia.

1 Centro de Implantes Cocleares Profesor Vicente Diamante, Pasteur 740, Buenos Aires. Argentina 


\section{INTRODUCCIÓN}

La pérdida auditiva bilateral severa a profunda, es aceptada como una de las causas más comunes de discapacidad en el ser humano; según diferentes reportes entre el 1 y $3 / 1.000$ nacidos vivos presentan hipoacusia severa a profunda por debajo de los 2 años de vida. La pérdida sensorial es producida en el $98 \%$ de los casos por alteraciones cocleares, y el resto de ellas por alteraciones del nervio auditivo o de la vía auditiva. Se debe intentar el diagnóstico y tratamiento o intervención temprana, la cual mejorará el pronóstico. Ouando la falla está en la cóclea en ausencia de patología del nervio auditivo y se acompaña de sordera severa a profunda, la indicación es de implante coclear (IC).

De manera tal que los pacientes que por diversas causas tenían una lesión o agenesia del nervio auditivo entre el ganglio espiral y los núcleos cocleares del tronco cerebral ( $4 \%$ de las hipoplasias del octavo par) no podían beneficiarse de una prótesis coclear y quedaban aislados en un mundo de silencio. En esos casos donde el nervio está lesionado no es posible la realización de un implante coclear y podría ser beneficiosa la utilización de un Implante Auditivo de Tronco Cerebral (IATC), que directamente estimula los núcleos cocleares (NC). Estos dispositivos están diseñados hasta ahora, para estimular los NC en su superficie y también en profundidad, con la posibilidad de proporcionar sensaciones auditivas a individuos con lesiones del nervio auditivo'.

\section{MATERIAL Y MÉTODO}

日 5 de marzo de 2007, el Prof. Diamante con la presencia del Prof. Colletti, realizaron en Buenos Aires, la primera cirugía para la colocación de un IATC en una niña de 2 años con agenesia de cóclea y de nervio. Este es el primer caso en toda América. $\mathrm{日} 18$ de abril de 2007, se colocó el IATC en otra niña de 10 años con agenesia de ambos nervios auditivos.

\section{Criterios de Indicación del IATC}

Según la FDA los candidatos al implante de tronco deben cumplir los siguientes criterios ${ }^{2,3}$ :
1. Diagnóstico de Neurofibromatosis tipo II (NPI),

2. Necesidad de remoción tumoral,

3. Ser mayor de 12 años,

4. Expectativas razonables.

\section{Compromiso de seguimiento}

Las nuevas indicaciones del implante de tronco en casos no tumorales para niños y adultos son ${ }^{4}$ :

1. Agenesia de cóclea,

2. Agenesia de nervio auditivo, 3

3. Grandes malformaciones de oído interno

4. Estallido traumático de ambas cócleas

5. Sección de ambos nervios auditivos

6. Cócleas osificadas posmeningitis con malos resultados con IC

7. Otoespongiosis masiva bilateral, otoesclerosis, osteogénesis imperfecta o enfermedad de Paget que hayan obtenido pobres resultados con IC

8. Neuronopatías auditivas que no respondieron a IC.

\section{RESULTADOS}

日 ABI permite la percepción de sonidos ambientales, monosílabos o bisílabos en formato cerrado. Un porcentaje reducido de pacientes llegan a la percepción en formato abierto. Todos ellos tienen una mejoría fundamental en la lectura labial. Vittorio Colletti, marca una diferencia significativa en relación a los resultados obtenidos con $A B I$ entre los pacientes con resección tumoral y los pacientes no tumorales. En los adultos no tumorales, el reconocimiento de palabras y monosílabos en formato cerrado al año, es de $86 \%$ y en los casos tumorales es de $24 \%$. Con respecto a la percepción de oraciones sin lectura labial, en casos no tumorales es de $66 \%$ al año y en los tumorales es de $20 \%$. Los niños prelinguales reconocen los bisílabos al año en 30\% en formato cerrado, y el $25 \%$ llega a la percepción de oraciones en formato abierto. De esta manera, si nos ajustamos a las indicaciones de la FDA no estaríamos ayudando a una serie de pacientes con patología no tumoral que pueden beneficiarse con el uso de un $\mathrm{ABI}$. Los resultados favorables obtenidos en los pacientes no tumorales en relación a los tumorales, se dan 


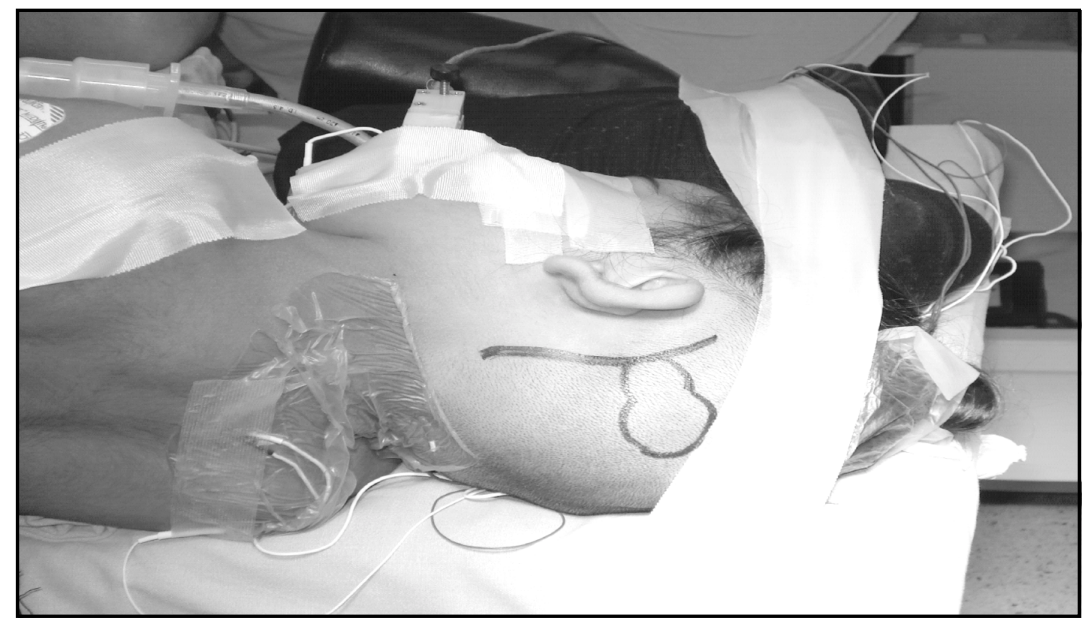

Fgura 1. Posición quirúrgica con la marca de las incisiones y del receptor interno.

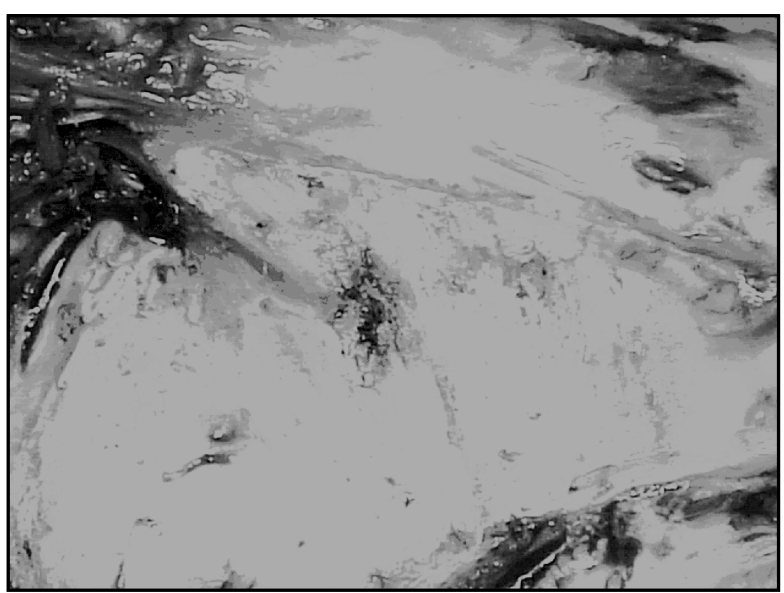

Figura 2. Apófisis mastoidea, límite posterior de la anura digástrica y zona anteroinferior del occipital.

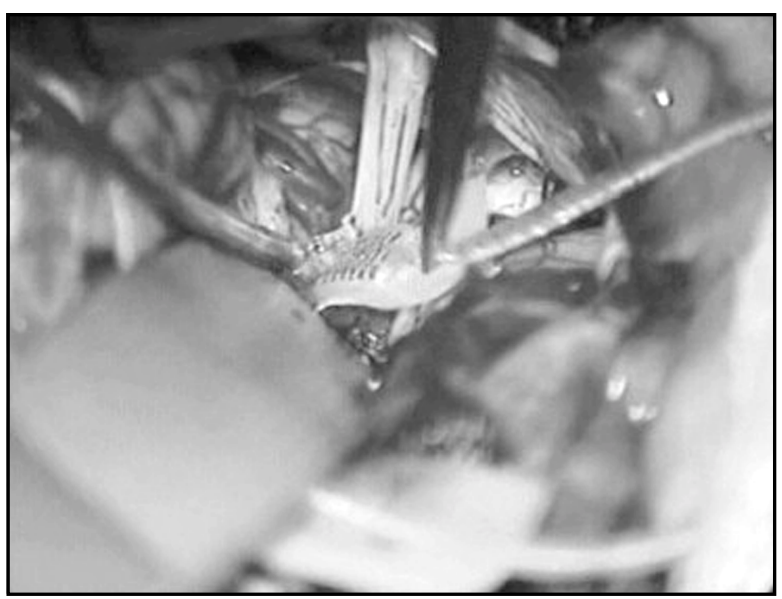

Figura 3. Nervio glosofaríngeo, plexos coroideos y electrodo. 
debido a que en los últimos, el tumor distorsiona la anatomía de los núcleos auditivos, comprimiendo y desplazando al tronco cerebral, dificultando a su vez la salida de LCR del receso lateral del IV ventrículo lo que produce edema, fibrosis e isquemia de las estructuras vecinas. Además, durante la cirugía de remoción tumoral se pueden producir, mediante la tracción y microcoagulación, interferencias en la vascularización de los núcleos. Según el Profesor Colleti, las diferencias pueden ser observadas durante la cirugía mediante las respuestas telemétricas, ya que en los casos tumorales se observan umbrales más altos que aquellos obtenidos en pacientes sin tumores, así como también existe un mayor número de electrodos activos con umbrales más estables en pacientes no tumorales.

Colletti, ha obtenido resultados significativos, en los casos de niños que presentaban grandes malformaciones cocleares y de osificaciones cocleares con falla en los resultados del IC que recibieron posteriormente un IATC.

Los resultados a los 7 días de nuestros dos casos fueron:

Caso 1: Paciente de 3 años

AT con IATC: entre 30db y $40 \mathrm{db}$, detecta todo el test de Ling (a-u-i-m-sh-s), comienza a identificar diferencias suprasegmentales (básicamente duración), muy buena y rápida respuesta al nombre en situación estructurada, aceptación total de la estimulación y por las mañanas solicita que le coloquen el implante (ver Figura 5).
Caso 2: Paciente de 10 años

AT con IATC: entre 35db y 40db, detecta todo el test de Ling (a-u-i-m-sh-s), identifica diferencias espectrales comienza a identificar palabras de igual duración por diferencias espectrales, responde al nombre con facilidad en situación estructurada, disfruta escuchar todos los ruidos ambientales (como agua, música, teléfono, etc.), no grita al hablar y no arrastra más los pies al caminar y usa el implante de forma permanente (ver Figura 6).

\section{DISCUSIÓN}

La FDA permite implantes auditivos de tronco cerebral solamente para pacientes con NFI mayores de 12 años. Para quienes presentan alteraciones intrínsecas de la cóclea que impide el IC o neuropatías de otras etiologías no está autorizada por dicha institución la colocación de IATC5.

Hace 5 años, el primer autor en el mundo en ampliar las indicaciones del IATC en niños y adultos no tumorales fue el Profesor Vittorio Colletti, de Verona, Italia $\mathrm{Si}$ sólo se realiza $\mathrm{ABI}$ en aquellos pacientes que sufren de sordera por NFI, siguiendo estrictamente las indicaciones de la FDA, se subestima la posibilidad de recuperación de los otros casos, lo que conlleva a que un número grande de individuos no puedan beneficiarse con un IATC, según el criterio de Colletti. A partir de ese concepto él comenzó a aplicar el uso del IATC en casos no tumorales adultos y niños ${ }^{6-10}$.

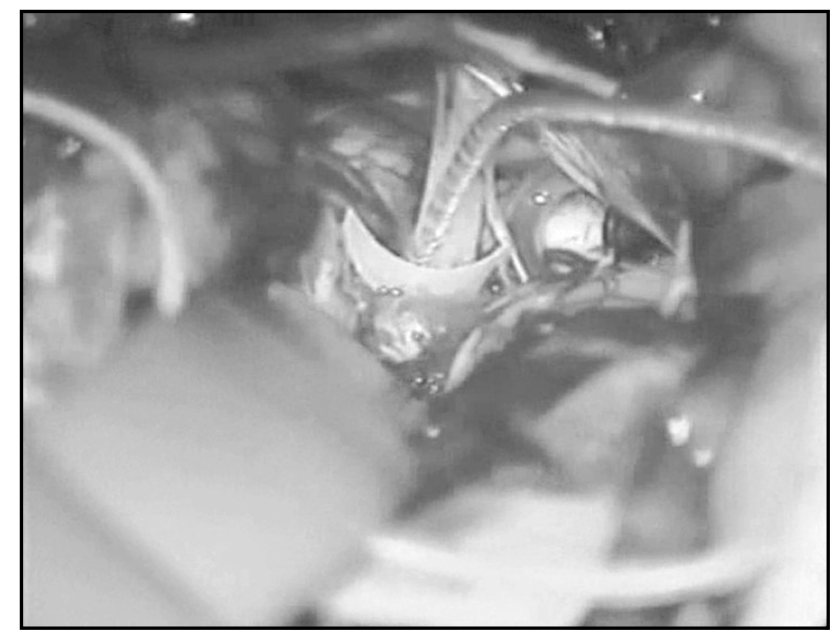

Figura 4. Posición definitiva del electrodo sostenido con músculo. 


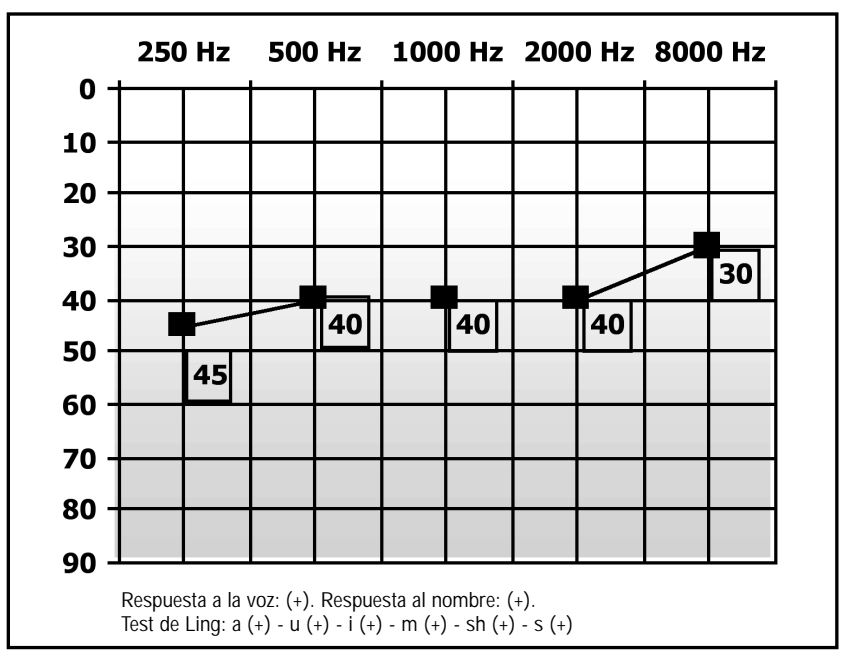

Figura 5. Resultados audiométricos del Caso 1.

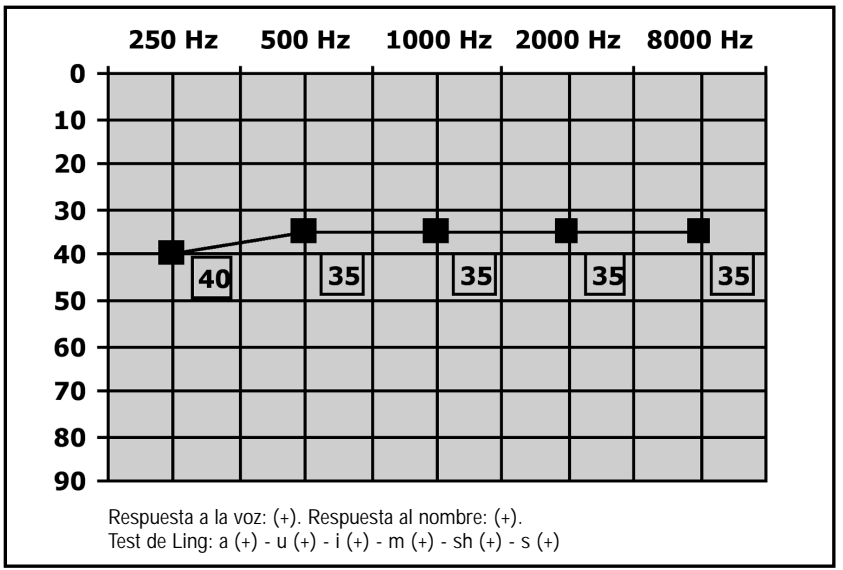

Figura 6. Resultados audiométricos del Caso 2.

日 IATC es hasta el momento la intervención más desafiante en el gran capítulo de la rehabilitación auditiva. En casos especiales, constituye la única posibilidad de realizar un by pass de la cóclea y el nervio auditivo, estimulando directamente la segunda neurona de la vía auditiva, estableciendo la conexión entre el mundo sonoro y el componente central de la vía auditiva, que hasta ese momento había estado totalmente privada de estimulación ${ }^{11}$.

La experiencia en la remoción de tumores en casos de NFI simultáneamente a la colocación de $\mathrm{ABI}$, durante 25 años, permite tener la certeza que la colocación del $\mathrm{ABI}$ no se asocia a un aumento de la morbilidad ni de las complicaciones posoperatorias. $\mathrm{日}$ hecho de saber que la estimulación directa de los núcleos del piso del IV ventrículo no producen alteraciones anatómicas ni funcionales de la vía auditiva, ni de las áreas vecinas, hace posible la idea de realizar el implante de tronco en niños desde muy temprana edad.

\section{CONCLUSIONES}

日 IATC ha sido considerado hasta hace pocos años sólo en los pacientes con NPI en especial cuando se realiza la cirugía para la extracción del neurinoma Actualmente, se está considerando en adultos y niños la utilización de este tipo de prótesis en pacientes con las causas no tumorales ya detalladas. 
日 grupo de IATCen niños consiste de profesionales de muchas disciplinas: otología, neurootología, audiología, psicología y terapia del habla y lenguaje.

日 impacto de este tipo de implantes en la vida de esos pacientes ha resultado muy positivo.

Los resultados observados son interesantes y aumentan nuestro conocimiento sobre las potencialidades del sistema auditivo central frente al estímulo eléctrico y las posibilidades de realización auditiva con la estimulación eléctrica en la superficie de los núcleos cocleares del tronco cerebral, haciendo un by-pass de la cóclea y el nervio.

\section{BIBLIOGRAFÍA}

1. Bamiou DE, Worth S, Phelps P, Sirimanna t, RAJPUT K. Egth nerve aplasia and hypoplasia in cochlear implant candidates: the clinical perspective. Atology \& Neurotology 2001; 22(4): 492-6.

2. Laszig $R$, Solmann WP, Marangos $N$. The restoration of hearing in Neurofibromatosis type 2. J Latyngol Otol 1995; 109: 385-9.

3. Lenarz M, Matthies C, Lesinski-Schimat A et al. Auditory brainstem implant Part II: subjective assessment of functional outcome. Otol Neurotol 2002; 23: 694-7.

4. Grayel aB, Bouocara D, Kalamarides M et al. Auditory brainstem implant in Bilateral and completely ossified cochleae. Otol Neurotol 2003; 24: 79-82.

5. Оाто SR, Brackman DE, Hitselberger WE Auditory brainstem implantation in 12-to 18 Years Ods. Arch Otolaryngol Head and Neck Surg 2004; 130: 656-9.

6. Coletm V, ForinoF, Carner M, SAochettoL, Miore山 V, ORSI A Auditory brainstem implantation: the University of Verona experience. Aolaryngol Head and Neck Surg 2002; 127: 84-96.

7. Collemt V, Forino F, Carner M et al. Hearing restoration with auditory brainstem implant in three children with cochlear nerve aplasia Otol Neurotol 2002; 23: 682-93.

8. Collett V, Forino F, Carngr M, Miorelu V, Gida M, Collem L. Auditory brainstem implant as a salvage treatment after unsuccessful cochlear implantation. Otol Neurotol 2004; 25: 485-96.

9. Collett V, Carner M, Miorell V, Gida M, Colsetr L, Forino F. Cochlear implant failure: Is an auditory brainstem implant the answer? Acta Otolaryngol 2004; 124: 353-57.

10. Collett V, Carner M, Miorell V, Gida M, Colletm L, Forino F. Auditory brainstem implant: new frontiers in adults and children. Otolaryngol Head and Neck Surg 2005; 133: 126-38.

11. McEieven JT JR, Hitsenberger WE House WF. Surgical accessibility of the cochlear nuclear complex in man: surgical landmarks. Aolaryngol Head and Neck Surg 1987; 96: 135-40. 